\title{
Quasinilpotent operators in operator Lie algebras II
}

\author{
by \\ Peng Cao (Beijing)
}

\begin{abstract}
In this paper, it is proved that the Banach algebra $\overline{\mathcal{A}(\mathcal{L})}$, generated by a Lie algebra $\mathcal{L}$ of operators, consists of quasinilpotent operators if $\mathcal{L}$ consists of quasinilpotent operators and $\overline{\mathcal{A}(\mathcal{L})}$ consists of polynomially compact operators. It is also proved that $\overline{\mathcal{A}(\mathcal{L})}$ consists of quasinilpotent operators if $\mathcal{L}$ is an essentially nilpotent Engel Lie algebra generated by quasinilpotent operators. Finally, Banach algebras generated by essentially nilpotent Lie algebras are shown to be compactly quasinilpotent.
\end{abstract}

1. Introduction. Let $\mathcal{X}$ be a Banach space, $\mathbf{B}(\mathcal{X})$ be the Banach algebra consisting of all bounded linear operators on $\mathcal{X}$, and $\mathbf{K}(\mathcal{X})$ be the ideal of compact operators. In $\mathbf{B}(\mathcal{X})$, we can define the Lie product [, ] : $\left[T_{1}, T_{2}\right]=T_{1} T_{2}-T_{2} T_{1}$ for any $T_{1}, T_{2} \in \mathbf{B}(\mathcal{X})$. So $\mathbf{B}(\mathcal{X})$ can be considered as a Lie algebra. For $M \subset \mathbf{B}(\mathcal{X})$, the Lie algebra generated by $M$, denoted by $\varepsilon(M)$, is the smallest Lie algebra containing $M$. Let $\mathcal{A}(M)$ denote the associative algebra generated by $M$, and $\bar{M}$ the closure of $M$ in $\mathbf{B}(\mathcal{X})$.

As usual, a compact and quasinilpotent operator is called a Volterra operator $\operatorname{Rad}(\mathcal{A})$ denotes the Jacobson radical of a Banach algebra $\mathcal{A}$.

Let $\mathcal{L}$ be a normed Lie algebra. A Banach Lie algebra is a complete normed Lie algebra. $\mathcal{L}$ is called an Engel Lie algebra if the adjoint operator ad $a: x \mapsto[a, x]$ is quasinilpotent for every $a \in \mathcal{L}$. Similarly, a normed algebra $\mathcal{A}$ is called an Engel algebra if the adjoint operator ad $a: x \mapsto[a, x]$ is quasinilpotent for every $a \in \mathcal{A}$. It is clear that nilpotent Lie algebras of operators are Engel Lie algebras.

To generalize the classical Engel theorem, W. Wojtyński posed the following question in [14]:

Question 1.1 (The generalized Engel theorem). Let $\mathcal{X}$ be a Banach space and $\mathcal{L}$ be a Banach Lie subalgebra of $\mathbf{B}(\mathcal{X})$ consisting of quasinilpotent

2010 Mathematics Subject Classification: Primary 47B07; Secondary 47L70.

Key words and phrases: Engel Lie algebras, essentially nilpotent Lie algebras, quasinilpotent operators. 
operators. Does the associative subalgebra of $\mathbf{B}(\mathcal{X})$ generated by $\mathcal{L}$ also consist of quasinilpotent operators?

One should note that there is a Lie algebra $\mathcal{L}$ consisting of nilpotent operators such that the Banach algebra $\overline{\mathcal{A}(\mathcal{L})}$ does not consist of quasinilpotent operators [4].

V. S. Shulman and Y. V. Turovskiı̌ have proved the following remarkable result (see [9, Theorem 11.4]):

Lemma 1.1. Suppose that a nonscalar Lie algebra $\mathcal{L} \subset \mathbf{B}(\mathcal{X})$ is the image of an Engel Banach Lie algebra under a bounded representation. If $\overline{\mathcal{L}}$ contains a nonzero compact operator, then $\mathcal{L}$ has a nontrivial hyperinvariant subspace.

As a corollary of Lemma 1.1, the following result is true (see [9, Corollaries 11.5 and 11.6]):

THEOREM 1.1. Let $\mathcal{L}$ be an Engel Lie algebra consisting of compact operators. The closed subalgebra of $\mathbf{B}(\mathcal{X})$ generated by $\mathcal{L}$ is commutative modulo the Jacobson radical. Moreover, any nonzero Lie algebra $\mathcal{L}$ of Volterra operators generates a Volterra algebra.

The following lemma will be used frequently (see [12]).

Lemma 1.2. Let $\mathcal{A}$ be a Banach algebra. If $\mathcal{L} \subset \mathcal{A}$ is a nilpotent Lie algebra, then the algebra $\overline{\mathcal{A}(\mathcal{L})} / \operatorname{Rad}(\overline{\mathcal{A}(\mathcal{L})})$ is commutative.

Lemma 1.3 ([6]). If $A / \operatorname{Rad}(A)$ is commutative, then $Q_{A}=\operatorname{Rad}(A)$, where $Q_{A}$ is the set of all quasinilpotent elements in the Banach algebra $A$.

In this paper, we will generalize Theorem 1.1 to noncompact operators.

2. Algebras consisting of polynomially compact operators. In this section, we will generalize Theorem 1.1 to the Banach algebra of polynomially compact operators.

An operator $T$ on an infinite-dimensional complex Banach space $\mathcal{X}$ is polynomially compact if there exists a nonzero complex polynomial $p$ such that the operator $p(T)$ is compact. If $T^{k}$ is compact for some $k$, we say that $T$ is a power compact operator. We refer to [7] for the basic properties of polynomially compact operators.

Let $\pi: \mathbf{B}(\mathcal{X}) \rightarrow \mathbf{B}(\mathcal{X}) / \mathbf{K}(\mathcal{X})$ be the natural homomorphism. A Lie algebra $\mathcal{L} \subset \mathbf{B}(\mathcal{X}) \mathcal{L}$ is called essentially nilpotent if $\pi(\mathcal{L})$ is nilpotent. The following result can be found in [2]:

Lemma 2.1. Let $\mathcal{L}$ be a Lie algebra of quasinilpotent operators on $\mathcal{X}$. If $\mathcal{L}$ is essentially nilpotent, then $\overline{\mathcal{A}(\mathcal{L})}$ consists of quasinilpotent operators. 
For the quotient mapping, we have the following result:

Lemma 2.2. Let $\overline{\mathcal{A}(\mathcal{L})}$ be the Banach algebra generated by a Lie algebra $\mathcal{L}, I$ be a closed ideal of $\overline{\mathcal{A}(\mathcal{L})}$, and $q: \overline{\mathcal{A}(\mathcal{L})} \rightarrow \overline{\mathcal{A}(\mathcal{L})} / I$ be the quotient mapping. Then $\overline{\mathcal{A}(q(\mathcal{L}))}=q(\overline{\mathcal{A}(\mathcal{L})})$.

Proof. It is clear that $\mathcal{A}(q(\mathcal{L}))=q(\mathcal{A}(\mathcal{L}))$. So $\overline{\mathcal{A}(q(\mathcal{L}))}=\overline{q(\mathcal{A}(\mathcal{L}))} \supset$ $q(\overline{\mathcal{A}(\mathcal{L})})$. Note that $q(\overline{\mathcal{A}(\mathcal{L})})$ is closed and contains $q(\mathcal{A}(\mathcal{L}))$. So $\overline{q(\mathcal{A}(\mathcal{L}))} \subset$ $q(\overline{\mathcal{A}(\mathcal{L})})$. Therefore, $\overline{\mathcal{A}(q(\mathcal{L}))}=\overline{q(\mathcal{A}(\mathcal{L}))}=q(\overline{\mathcal{A}(\mathcal{L})})$.

The following result may be known, but we cannot find the exact reference.

Proposition 2.1. If each element of a Banach algebra $\mathcal{B}$ is algebraic, then $\mathcal{B} / \operatorname{Rad}(\mathcal{B})$ is finite-dimensional and $\operatorname{Rad}(\mathcal{B})$ is nilpotent.

Proof. Since each element of $\mathcal{B}$ is algebraic, it has finite spectrum. By Theorem 5.4.2 in [1], $\mathcal{B} / \operatorname{Rad}(\mathcal{B})$ is finite-dimensional. For any $T \in \operatorname{Rad}(\mathcal{B})$, $T$ is quasinilpotent and algebraic, so $T$ is a nilpotent operator. That is, $\operatorname{Rad}(\mathcal{B})$ consists of nilpotent operators. By Grabiner's result $([5]), \operatorname{Rad}(\mathcal{B})$ is nilpotent.

Now we give the main result of this section. Recall that if a finitedimensional Lie algebra consists of quasinilpotent operators, then by Rosenblum's theorem and Engel's theorem, it is nilpotent.

THEOREM 2.1. Let $\mathcal{L}$ consist of quasinilpotent operators, and suppose that $\overline{\mathcal{A}(\mathcal{L})}$ consists of polynomially compact operators. Then $\overline{\mathcal{A}(\mathcal{L})}$ consists of quasinilpotent operators.

Proof. First, we will prove that $\overline{\mathcal{A}(\mathcal{L})}$ consists of power compact operators.

Let $I=\overline{\mathcal{A}(\mathcal{L})} \cap \mathbf{K}(\mathcal{X})$. Then $I$ is a closed ideal of $\overline{\mathcal{A}(\mathcal{L})}$. Let $q: \overline{\mathcal{A}(\mathcal{L})} \rightarrow$ $\overline{\mathcal{A}(\mathcal{L})} / I$ be the quotient mapping. Then $\mathcal{B}:=q(\overline{\mathcal{A}(\mathcal{L})})$ is a Banach algebra. For every $[T] \in \mathcal{B}$, there is an operator $T$ in $\overline{\mathcal{A}(\mathcal{L})}$ such that $[T]=q(T)$. Because $T$ is polynomially compact by assumption, there is a polynomial $p$ such that $p(T) \in \mathbf{K}(\mathcal{X})$. Let $f(T):=p(T) T$. Then $f(T) \in \mathbf{K}(\mathcal{X})$, and $f(T) \in$ $\overline{\mathcal{A}(\mathcal{L})}$ because $f(0)=0$. Hence $f(T) \in \mathbf{K}(\mathcal{X}) \cap \overline{\mathcal{A}(\mathcal{L})}=I$. Therefore, $f([T])=$ $q(f(T))=0$. That is, every element in $\mathcal{B}$ is algebraic. By Proposition 2.1, $\mathcal{B} / \operatorname{Rad}(\mathcal{B})$ is finite-dimensional. Let $\gamma: \mathcal{B} \rightarrow \mathcal{B} / \operatorname{Rad}(\mathcal{B})$ be the natural homomorphism. By Lemma 2.2 , it is clear that $\mathcal{B}$ is the Banach algebra generated by $q(\mathcal{L})$, and $\mathcal{B} / \operatorname{Rad}(\mathcal{B})$ is the Banach algebra generated by the Lie algebra $\gamma(q(\mathcal{L}))$. For every $Q \in \mathcal{L}$, since $Q$ is quasinilpotent, so is $\gamma(q(Q))$ in $\mathcal{B} / \operatorname{Rad}(\mathcal{B})$. Note that $\mathcal{B} / \operatorname{Rad}(\mathcal{B})$ is finite-dimensional, so $\gamma(q(\mathcal{L}))$ is a finitedimensional Lie algebra consisting of quasinilpotent elements. Hence $\gamma(q(\mathcal{L}))$ is nilpotent. By Lemmas 1.2 and $1.3, \mathcal{B} / \operatorname{Rad}(\mathcal{B})$ consists of quasinilpotent elements. Thus $\mathcal{B} / \operatorname{Rad}(\mathcal{B})=\operatorname{Rad}(\mathcal{B} / \operatorname{Rad}(\mathcal{B})) . \operatorname{But} \operatorname{Rad}(\mathcal{B} / \operatorname{Rad}(\mathcal{B}))=\{0\}$. 
So $\mathcal{B}=\operatorname{Rad}(\mathcal{B})$. Consequently, $\mathcal{B}$ consists of quasinilpotent elements. Note that every element in $\mathcal{B}$ is algebraic. So $\mathcal{B}$ consists of nilpotent elements. That is, $\overline{\mathcal{A}(\mathcal{L})}$ consists of power compact operators.

Since $\mathcal{B}=\operatorname{Rad}(\mathcal{B})$ consists of nilpotent elements, $\mathcal{B}$ is nilpotent as an associative algebra by Proposition 2.1. So $\mathcal{B}$ is nilpotent as a Lie algebra. Therefore, $\mathcal{L}$ is essentially nilpotent. By Lemma $2.1, \overline{\mathcal{A}(\mathcal{L})}$ consists of quasinilpotent operators.

REMARK 2.1. It is easy to see that if $\mathcal{L}$ consists of quasinilpotent operators which are power compact with uniform index (that is, there exists some $n \in \mathbb{N}$ such that $T^{n}$ is compact for every $\left.T \in \mathcal{L}\right)$, then $\overline{\mathcal{A}(\mathcal{L})}$ consists of quasinilpotent operators. In fact, $\mathcal{L}$ is then essentially nilpotent, and $\overline{\mathcal{A}(\mathcal{L})}$ consists of quasinilpotent operators by Lemma 2.1.

3. Essentially nilpotent Engel Lie algebras. In this section, we generalize Theorem 1.1 to essentially nilpotent Engel Banach algebras.

For $I \subset \mathbf{B}(\mathcal{X})$, let Lat $I$ be the set of all invariant subspaces of $I$. Let $\Pi \subset$ Lat $I$. We say that $V$ is a gap-quotient of $\Pi$ if $V=Y / Z$, where $Y, Z \in \Pi, Z \subset Y$ and there exist no subspaces in $\Pi$ which are intermediate between $Y$ and $Z$. For every $T \in I,\left.T\right|_{V}$ denotes the operator induced by $T$ on $V$, and $\left.M\right|_{V}:=\left\{\left.T\right|_{V} \mid T \in M\right\}$ for $M \subset I$.

Lemma 3.1 ([10, Lemma 2.6]). Let $\mathcal{M} \subset \mathbf{K}(\mathcal{X}), N \subset \mathbf{B}(\mathcal{X})$ and suppose $\mathcal{M} \subset N$. The following conditions are equivalent:

(i) $\left.\mathcal{M}\right|_{V}=0$ for any gap-quotient $V$ of Lat $N$.

(ii) $\mathcal{M} \subset \operatorname{Rad}(\overline{\mathcal{A}(N)})$.

If $\mathcal{L}$ is a Lie algebra, let $\mathcal{L}^{(1)}=\mathcal{L}$ and $\mathcal{L}^{(k)}=\left[\mathcal{L}, \mathcal{L}^{(k-1)}\right]$ for $k \geq 2$.

Recall that a Riesz operator is an operator $T$ in $\mathbf{B}(\mathcal{X})$ such that $\pi(T)$ is quasinilpotent in $\mathbf{B}(\mathcal{X}) / \mathbf{K}(\mathcal{X})$. If $Q$ is a quasinilpotent operator and $V$ is a gap-quotient of Lat $Q$, then $\left.Q\right|_{V}$ is also a quasinilpotent operator on $V$.

For a Lie algebra $\mathcal{L} \subset \mathbf{B}(\mathcal{X}), \overline{\mathcal{L}}$ means the closure of $\mathcal{L}$ in $\mathbf{B}(\mathcal{X})$.

For any $T \in \mathcal{L}, \operatorname{ad} T: \mathcal{L} \rightarrow \mathcal{L}$ is quasinilpotent (i.e., $\left.\lim \left\|(\operatorname{ad} T)^{n}\right\|^{1 / n}=0\right)$ if and only if $\operatorname{ad} T: \overline{\mathcal{L}} \rightarrow \overline{\mathcal{L}}$ is quasinilpotent if and only if $\left\|(\operatorname{ad} T)^{n}(S)\right\|^{1 / n}$ $\rightarrow 0$, for any $S \in \overline{\mathcal{L}}$, as $n \rightarrow \infty$.

For any $S \in \mathbf{B}(\mathcal{X}), \lambda \in \mathbb{C}$ and $r \geq 0$, the elementary spectral manifold $\eta_{\lambda, r}(S)$ of $S$ is defined as follows (see [10]:

$$
\eta_{\lambda, r}(S):=\left\{x \in X \mid \limsup \left\|(S-\lambda)^{n} x\right\|^{1 / n} \leq r\right\}
$$

and

$$
\eta_{\lambda}(S):=\eta_{\lambda, 0}(S) .
$$

Some results on elementary spectral manifolds can be found in Section 3 of $[10]$. 
THEOREM 3.1. Let $\mathcal{L}$ be a Lie algebra generated by quasinilpotent operators. If $\mathcal{L}$ is Engel and essentially nilpotent, then $\overline{\mathcal{A}(\mathcal{L})}$ consists of quasinilpotent operators.

Proof. Let $\mathcal{L}=\varepsilon(\mathcal{Q})$, where $\mathcal{Q}$ consists of quasinilpotent operators. It is easy to see that $\overline{\mathcal{A}(\mathcal{L})}=\overline{\mathcal{A}(\mathcal{Q})}$, and $\operatorname{Lat}\{\mathcal{Q}\}=\operatorname{Lat}\{\mathcal{L}\}=\operatorname{Lat}\{\overline{\mathcal{A}(\mathcal{L})}\}$.

Let $\pi: \mathbf{B}(\mathcal{X}) \rightarrow \mathbf{B}(\mathcal{X}) / \mathbf{K}(\mathcal{X})$ be the natural homomorphism. Since $\pi(\mathcal{L})$ is a nilpotent Lie algebra, there is an integer $k$ such that $\pi\left(\mathcal{L}^{(k)}\right)=$ $(\pi(\mathcal{L}))^{(k)}=\{0\}$. If $\mathcal{L}^{(k)}=\{0\}$, then $\mathcal{L}$ is nilpotent. By Lemmas 1.2 and 1.3, $\overline{\mathcal{A}(\mathcal{L})}$ consists of quasinilpotent operators.

If $\mathcal{L}^{(k)} \neq\{0\}$, then there is a nonzero compact operator in $\mathcal{L}^{(k)}$. If $k=1$, that is, $\pi(\mathcal{L})=0$, then $\mathcal{L}$ consists of compact operators, and the result follows by Theorem 1.1 and Lemmas 1.2 and 1.3. So we assume that $k>1$.

Claim 1. For any gap-quotient $V$ of Lat $\mathcal{L}, \overline{\mathcal{A}\left(\left.\mathcal{L}\right|_{V}\right)}$ consists of Riesz operators on $V$.

For any gap-quotient $V$ of Lat $\mathcal{L},\left.\mathcal{Q}\right|_{V}$ consists of quasinilpotent operators on $V$ and $\left.\mathcal{L}^{(k)}\right|_{V}$ consists of compact operators on $V$. Let $q: \mathbf{B}(V) \rightarrow$ $\mathbf{B}(V) / \mathbf{K}(V)$ be the natural homomorphism. As $q(\mathcal{L})$ is a nilpotent Lie algebra, $\overline{\mathcal{A}\left(q\left(\left.\mathcal{L}\right|_{V}\right)\right)}=\overline{\mathcal{A}\left(q\left(\left.\mathcal{Q}\right|_{V}\right)\right)}$ consists of quasinilpotent operators by Lemmas 1.2 and 1.3. So $\overline{\mathcal{A}\left(\left.\mathcal{L}\right|_{V}\right)}$ consists of Riesz operators on $V$.

Claim 2. $\left.\mathcal{L}\right|_{V}$ is an Engel Lie algebra on $V$.

For every $S \in \mathcal{L}, \lim \left\|(\operatorname{ad} S)^{n}(T)\right\|^{1 / n}=0$ for any $T \in \mathcal{L}$, because $\mathcal{L}$ is Engel. Therefore

$$
\lim \|\left(\left(\left.\operatorname{ad} S\right|_{V}\right)^{n}\left(\left.T\right|_{V}\right)\left\|^{1 / n} \leq \lim \right\|(\operatorname{ad} S)^{n}(T) \|^{1 / n}=0 .\right.
$$

Hence $\left.\mathcal{L}\right|_{V} \subset \eta_{0}\left(\left.\operatorname{ad} S\right|_{V}\right)$. Note that $\left.S\right|_{V}$ is a Riesz operator. ad $\left.S\right|_{V}$ has countable spectrum by Rosenblum's theorem. By Proposition 3.3(i) in [10], $\eta_{0}\left(\left.\operatorname{ad} S\right|_{V}\right)$ is closed. So $\overline{\left.\mathcal{L}\right|_{V}} \in \eta_{0}\left(\left.\operatorname{ad} S\right|_{V}\right)$. ad $\left.S\right|_{V}: \overline{\left.\mathcal{L}\right|_{V}} \rightarrow \overline{\left.\mathcal{L}\right|_{V}}$ is quasinilpotent by Proposition 3.3(ii) in [10]. That is, $\left.\mathcal{L}\right|_{V}$ is an Engel Lie algebra on $V$.

Claim 3. $\overline{\left.\mathcal{L}\right|_{V}}$ is an Engel Lie algebra on $V$.

For every $\left.S\right|_{V} \in \overline{\left.\mathcal{L}\right|_{V}}$, there is a sequence $\left\{\left.T_{n}\right|_{V}\right\}$ in $\left.\mathcal{L}\right|_{V}$ such that $\left.\lim _{n \rightarrow \infty} T_{n}\right|_{V}=\left.S\right|_{V}$, and $\left.S\right|_{V}$ is a Riesz operator by Claim 1. The spectrum of $\left.S\right|_{V}$ is countable. So the spectrum of ad $\left.S\right|_{V}$ is countable by Rosenblum's theorem. Note that $\lim _{n \rightarrow \infty}$ ad $\left.T_{n}\right|_{V}=\left.\operatorname{ad} S\right|_{V}$, and the spectral radius is continuous at ad $\left.S\right|_{V}$ (cf.[8]). We have $r\left(\left.\operatorname{ad} T_{n}\right|_{V}\right)=0$ on $\overline{\left.\mathcal{L}\right|_{V}}$ since the operator ad $\left.T_{n}\right|_{V}: \overline{\left.\mathcal{L}\right|_{V}} \rightarrow \overline{\left.\mathcal{L}\right|_{V}}$ is quasinilpotent by Claim 2, so $r\left(\left.\operatorname{ad} S\right|_{V}\right)=0$. Hence, $\overline{\left.\mathcal{L}\right|_{V}}$ is an Engel Lie algebra.

Claim 4. Every operator in $\mathcal{L}^{(k)}$ is in $\operatorname{Rad}(\overline{\mathcal{A}(\mathcal{L})})$.

Note $\overline{\left.\mathcal{L}\right|_{V}}$ is a closed Engel Lie algebra in $\mathbf{B}(V)$. For every $T \in \mathcal{L}^{(k)}, T$ is a compact operator, and hence so is $\left.T\right|_{V}$. Suppose $\left.T\right|_{V} \neq 0$. If $\operatorname{dim} V \neq 1$, then 
by Lemma 1.1, $\left.\mathcal{L}\right|_{V}$ has a nontrivial invariant subspace. This contradicts the choice of $V$. So $\operatorname{dim} V=1$. But for every $R_{1}, R_{2} \in \mathcal{L}$, since $\operatorname{dim} V=1$, we have $\left.\left[R_{1}, R_{2}\right]\right|_{V}=0$. Note $T \in \mathcal{L}^{(k)}=\left[\mathcal{L}, \mathcal{L}^{(k-1)}\right]$, so $\left.T\right|_{V}=0$. This is also a contradiction. So $\left.T\right|_{V}=0$. By Lemma 3.1, $T \in \operatorname{Rad}(\overline{\mathcal{A}(\mathcal{L})})$.

Let $\gamma$ be the canonical morphism $\overline{\mathcal{A}(\mathcal{L})} \rightarrow \overline{\mathcal{A}(\mathcal{L})} / \operatorname{Rad}(\overline{\mathcal{A}(\mathcal{L})})$. Then $\gamma(\mathcal{L})$ is nilpotent. By Lemma 2.2, the Banach algebra generated by $\gamma(\mathcal{L})$ is $\overline{\mathcal{A}(\mathcal{L})} / \operatorname{Rad}(\overline{\mathcal{A}(\mathcal{L})})$. So

$$
[\overline{\mathcal{A}(\gamma(\mathcal{L}))}, \overline{\mathcal{A}(\gamma(\mathcal{L}))}] \subset \operatorname{Rad}(\overline{\mathcal{A}(\mathcal{L})} / \operatorname{Rad}(\overline{\mathcal{A}(\mathcal{L})}))=\{0\}
$$

by Lemma 1.2. Therefore, $\gamma([\overline{\mathcal{A}(\mathcal{L})}, \overline{\mathcal{A}(\mathcal{L})}]) \subset[\overline{\mathcal{A}(\gamma(\mathcal{L}))}, \overline{\mathcal{A}(\gamma(\mathcal{L}))}]=\{0\}$. That is, $\overline{\mathcal{A}(\mathcal{L})}$ is commutative modulo the Jacobson radical. So $\overline{\mathcal{A}(\mathcal{L})}$ consists of quasinilpotent operators by Lemma 1.3.

4. Compact quasinilpotence. The theory of topological radicals of normed algebras was initiated by P. G. Dixon [3]. The definition of compact quasinilpotence was given by V. S. Shulman and Y. V. Turovskiu [11]. One can find the definition of joint spectral radical in [9].

Definition 4.1. A normed algebra $\mathcal{A}$ is compactly quasinilpotent if $\rho(M)=0$ for every precompact set $M \subset \mathcal{A}$, where $\rho(M)$ is the joint spectral radical of $M$.

Define

$$
R_{c}(\mathcal{A}):=\{a \in \mathcal{A} \mid \rho(\{a\} \cup M)=\rho(M)
$$

for every precompact set $M \subset \mathcal{A}\}$.

$R_{c}(\mathcal{A})$ is called the compactly quasinilpotent radical. $R_{c}(\mathcal{A})$ is compactly quasinilpotent ([11, Proposition 4.13]). For details, see Section 4 in [11].

The following lemma can be found in [13].

Lemma 4.1. If a Banach algebra $\mathcal{A}$ is generated by a nilpotent Lie subalgebra or a finite-dimensional solvable Lie subalgebra, then $\mathcal{A} / R_{c}(\mathcal{A})$ is commutative, and $R_{c}(\mathcal{A})=\operatorname{Rad}(\mathcal{A})$.

THEOREM 4.1. Let $\mathcal{L}$ be a Lie algebra of quasinilpotent operators on $\mathcal{X}$. If $\mathcal{L}$ is an essentially nilpotent Lie algebra, then $\overline{\mathcal{A}(\mathcal{L})}$ is compactly quasinilpotent.

Proof. By Lemma 2.1, $\overline{\mathcal{A}(\mathcal{L})}$ consists of quasinilpotent operators. Since $\pi(\mathcal{L})$ is a nilpotent Lie algebra, there exists an integer $k$ such that $\pi\left(\mathcal{L}^{(k)}\right)=$ $(\pi(\mathcal{L}))^{(k)}=\{0\}$. If $\mathcal{L}^{(k)}=\{0\}$, then $\mathcal{L}$ is nilpotent. By Lemma 4.1, $R_{c}(\overline{\mathcal{A}(\mathcal{L})})$ $=\operatorname{Rad}(\overline{\mathcal{A}(\mathcal{L})})=\overline{\mathcal{A}(\mathcal{L})}$. By Proposition 4.13 in [11], $\overline{\mathcal{A}(\mathcal{L})}$ is compactly quasinilpotent.

If $\mathcal{L}^{(k)} \neq\{0\}$, then $\mathcal{L}^{(k)}$ consists of Volterra operators. For every $a \in \mathcal{L}^{(k)}$ and every precompact set $M \subset \overline{\mathcal{A}(\mathcal{L})}, a M$ consists of Volterra operators. 
Note that $\overline{\mathcal{A}(a M)}$ consists of Volterra operators, because $\overline{\mathcal{A}(\mathcal{L})}$ consists of quasinilpotent operators. So by Corollary 4.9 in [9], $\overline{\mathcal{A}(a M)}$ is compactly quasinilpotent. Then $\rho(a M)=0$, because $a M$ is a precompact set in $\overline{\mathcal{A}(a M)}$. By Theorem 4.20 in [11], $a \in R_{c}(\overline{\mathcal{A}(\mathcal{L})})$. Therefore, $\mathcal{L}^{(k)} \subset R_{c}(\overline{\mathcal{A}(\mathcal{L})})$. Let $\mathcal{B}=\overline{\mathcal{A}(\mathcal{L})} / R_{c}(\overline{\mathcal{A}(\mathcal{L})})$. Since $R_{c}(\overline{\mathcal{A}(\mathcal{L})})$ is a closed ideal ([11, Theorem 4.10$\left.]\right)$, $\mathcal{B}$ is a Banach algebra. By Lemma $2.2, \mathcal{B}$ is generated by $\mathcal{L} / R_{c}(\overline{\mathcal{A}(\mathcal{L})})$. Note that $\mathcal{L} / R_{c}(\overline{\mathcal{A}(\mathcal{L})})$ is nilpotent, so by Lemma $4.1, R_{c}(\mathcal{B})=\operatorname{Rad}(\mathcal{B})=\mathcal{B}$. But by Lemma 4.23 in [11],

$$
R_{c}(\mathcal{B})=R_{c}\left(\overline{\mathcal{A}(\mathcal{L})} / R_{c}(\overline{\mathcal{A}(\mathcal{L})})\right)=\{0\} .
$$

So $\mathcal{B}=\{0\}$, and $\overline{\mathcal{A}(\mathcal{L})}=R_{c}(\overline{\mathcal{A}(\mathcal{L})})$. By Proposition 4.13 in $[11], \overline{\mathcal{A}(\mathcal{L})}$ is compactly quasinilpotent.

COROLlary 4.1. Let $\mathcal{L}$ consist of quasinilpotent operators, and suppose that $\overline{\mathcal{A}(\mathcal{L})}$ consists of polynomially compact operators. Then $\overline{\mathcal{A}(\mathcal{L})}$ is compactly quasinilpotent.

Proof. It is known from the proof of Theorem 2.1 that $\mathcal{L}$ is essentially nilpotent. The result holds by Theorem 4.1.

COROLlary 4.2. Let $\mathcal{L}$ be a Lie algebra generated by quasinilpotent operators. If $\mathcal{L}$ is Engel and essentially nilpotent, then $\overline{\mathcal{A}(\mathcal{L})}$ is compactly quasinilpotent.

Proof. This is trivial by Theorems 3.1 and 4.1.

Acknowledgements. The author would like to express his gratitude to Y. V. Turovskiü, V. S. Shulman, and P. G. Dixon for sending their papers. He also thanks the referee for useful suggestions.

This project was supported by "National Natural Science Foundation of China No. 10601005, No. 10801008" and "Excellent Young Scholars Research Fund of Beijing Institute of Technology".

\section{References}

[1] B. Aupetit, A Primer on Spectral Theory, Springer, New York, 1991.

[2] P. Cao and S. Sun, Quasinilpotent operators in operator Lie algebras, Integral Equations Operator Theory 58 (2007), 35-41.

[3] P. G. Dixon, Topologically irreducible representations and radicals in Banach algebras, Proc. London Math. Soc. (3) 74 (1997), 174-200.

[4] D. Hadwin, E. Nordgren, M. Radjabalipour, H. Radjavi, and P. Rosenthal, A nil algebra of operators on Hilbert spaces with semisimple norm closure, Integral Equations Operator Theory 9 (1986), 729-743.

[5] S. Grabiner, The nilpotency of Banach nil algebras, Proc. Amer. Math. Soc. 21 (1969), 510.

[6] A. Katavolos and C. Stamatopoulos, Commutators of quasinilpotents and invariant subspaces, Studia Math. 128 (1998), 159-169. 
[7] M. Konvalinka, Triangularizability of polynomially compact operators, Integral Equations Operator Theory 52 (2005), 271-284.

[8] J. Newburgh, The variation of spectra, Duke Math. J. 18 (1951), 165-176.

[9] V. S. Shulman and Y. V. Turovskiur, Joint spectral radius, operator semigroups, and a problem of W. Wojtyński, J. Funct. Anal. 177 (2000), 383-441.

[10] - - - Invariant subspaces of operator Lie algebras and Lie algebras with compact adjoint action, ibid. 223 (2005), 425-508.

[11] —, - Topological radicals I, in: Topological Algebras, Their Applications, and Related Topics, Banach Center Publ. 67, Inst. Math., Polish Acad. Sci., 2005, 293333.

[12] Y. V. Turovskiur, Spectral properties of certain Lie subalgebras and the spectral radius of subsets of a Banach algebra, in: Spectral Theory of Operators and its Applications, F. G. Maksudov (ed.), Vol. 6, Elm, Baku, 1985, 144-181 (in Russian).

[13] Y. V. Turovskiu and V. S. Shulman, Radicals in Banach algebras and some problems in the theory of radical Banach algebras, Funct. Anal. Appl. 35 (2001), 312-314.

[14] W. Wojtyński, Quasinilpotent Banach-Lie algebras are Baker-Campbell-Hausdorff, J. Funct. Anal. 153 (1998), 405-413.

Department of Mathematics

Beijing Institute of Technology

Beijing, China, 100081

E-mail: cpeng001@163.com

Received January 1, 2009

Revised version June 22, 2009 